\title{
When to perform positron emission tomography/computed tomography or radionuclide bone scan in patients with recently diagnosed prostate cancer
}

\author{
This article was published in the following Dove Press journal: \\ Cancer Management and Research \\ 24 June 2013 \\ Number of times this article has been viewed
}

\section{Carmelo Caldarella' \\ Giorgio Treglia ${ }^{2}$ \\ Alessandro Giordano' \\ Luca Giovanella ${ }^{2}$ \\ 'Institute of Nuclear Medicine, Catholic University of the Sacred Heart, Rome, Italy; ${ }^{2}$ Department of Nuclear Medicine and PET/CT Centre, Oncology Institute of Southern Switzerland, Bellinzona, Switzerland}

Correspondence: Carmelo Caldarella Institute of Nuclear Medicine, Catholic University of the Sacred Heart, 8 Largo Agostino Gemelli,

Rome 00168 , Italy

Tel +390630156200

Fax +39063013745

Email carmelocaldarella@yahoo.it

\begin{abstract}
Skeletal metastases are very common in prostate cancer and represent the main metastatic site in about $80 \%$ of prostate cancer patients, with a significant impact in patients' prognosis. Early detection of bone metastases is critical in the management of patients with recently diagnosed high-risk prostate cancer: radical treatment is recommended in case of localized disease; systemic therapy should be preferred in patients with distant secondary disease. Bone scintigraphy using radiolabeled bisphosphonates is of great importance in the management of these patients; however, its main drawback is its low overall accuracy, due to the nonspecific uptake in sites of increased bone turnover. Positron-emitting radiopharmaceuticals, such as fluorine-18-fluorodeoxyglucose, choline-derived drugs (fluorine-18-fluorocholine and carbon-11-choline) and sodium fluorine-18-fluoride, are increasingly used in clinical practice to detect metastatic spread, and particularly bone involvement, in patients with prostate cancer, to reinforce or substitute information provided by bone scan. Each radiopharmaceutical has a specific mechanism of uptake; therefore, diagnostic performances may differ from one radiopharmaceutical to another on the same lesions, as demonstrated in the literature, with variable sensitivity, specificity, and overall accuracy values in the same patients. Whether bone scintigraphy can be substituted by these new methods is a matter of debate. However, greater radiobiological burden, higher costs, and the necessity of an in-site cyclotron limit the use of these positron emission tomography methods as first-line investigations in patients with prostate cancer: bone scintigraphy remains the mainstay for the detection of bone metastases in current clinical practice.
\end{abstract}

Keywords: bone metastases, prostate cancer, bisphosphonates, positron emission tomography

\section{Introduction}

Prostate cancer is the most common malignancy in men, especially in the elderly: this condition is more frequently diagnosed over age 65 years, although extensive screening programs through widespread availability of prostate-specific antigen (PSA) assay have increased the possibility of diagnosis at an earlier age (40-50 years). ${ }^{1-3}$ Skeletal metastases are very common in prostate cancer, with an $84 \%$ prevalence on autoptic series, and represent the initial and main metastatic site in about $80 \%$ of prostate cancer patients. ${ }^{4}$ Bone involvement significantly affects the prognosis of these patients; particularly, the rate of increase in the number of bone metastases, as detected on bone scintigraphy, is associated with lower survival rates after the detection of bone involvement itself. ${ }^{5}$ Several studies have demonstrated that the 
extent of bone involvement from prostate cancer is an independent negative prognostic factor. ${ }^{6-8}$ The incidence of bone metastases depends on many factors: it is reasonably low in patients with Gleason score (GS) of the primary tumor lower than 6 and PSA lower than $20 \mathrm{ng} / \mathrm{mL} ;{ }^{9}$ however, Moslehi et $\mathrm{al}^{10}$ found that a combination of elevated PSA $(>20 \mathrm{ng} / \mathrm{mL})$ and serum alkaline phosphatase, regardless of GS, best predicts the risk of bone metastases in asymptomatic patients with newly diagnosed prostate cancer, with $98.2 \%$ sensitivity.

Common sites of bone metastases from prostate cancer are vertebral bodies, sternum, pelvic bones, ribs, and proximal femurs; skull base and orbital and extremity involvement is fairly uncommon. ${ }^{11,12}$ However, pathological fractures are unusual, because metastases from prostate cancer usually show a marked osteoblastic reaction of the surrounding bone. Bone pain and spinal cord compression are more frequently seen; most cases are asymptomatic.

Early detection of metastatic bone involvement is critical in the management of patients with high-risk prostate cancer, since radical treatment with curative intent (surgery, radiation therapy, or both) is recommended only in patients presenting with localized disease; patients with metastatic disease should be treated with systemic therapy (androgen-deprivation therapy, bisphosphonates). ${ }^{13-15}$ Moreover, accurate staging could be helpful in identifying patients with single-bone metastasis susceptible to radiation therapy, particularly when painful.

Radionuclide bone scintigraphy is the most widely used nuclear medicine diagnostic technique in evaluating patients with high-risk prostate cancer and suspicion of skeletal metastases: it is highly accurate, available in almost all nuclear medicine units, and is simple to execute. However, new diagnostic methods such as positron emission tomography (PET)/computed tomography (CT) are increasingly used for this purpose, in order to make the identification of bone metastases easier and to overcome the limitations of bone scintigraphy (low specificity). This notwithstanding, whether and when these PET/CT techniques should be performed in these patients, or even whether they could substitute bone scintigraphy in the future, is still a matter of debate.

The aim of this review was to investigate the state of the art of published literature about $\mathrm{PET} / \mathrm{CT}$ and radionuclide bone scans to define what the contribution of each of these techniques is in the management of patients with recent diagnosis of prostate cancer, and therefore when they should be performed.

\section{Radionuclide bone scintigraphy}

Radionuclide bone scintigraphy using radiolabeled bisphosphonates is the mainstay for the detection of skeletal metastases in patients with various tumoral entities. Bisphosphonates are organic phosphate compounds currently used as therapeutic agents for osteoporosis, due to their ability to reduce bone resorption by inhibiting human osteoblast secretion and proliferation. ${ }^{16}$ The rationale of using bisphosphonates for the detection of bone malignancies is that the presence of neoplastic cells usually induces increased turnover by the surrounding bone tissue; therefore, bisphosphonates primarily accumulate on the surface of crystals of hydroxyapatite around neoplastic tissue, rather than in the healthy bone. Technetium-99m methylene bisphosphonate (MDP) and technetium-99m hydroxymethylene bisphosphonate (HMDP) are the radiopharmaceuticals of choice to detect skeletal metastases in patients with high-risk prostate cancer at either first diagnosis or suspicion of relapse due to doubtful imaging findings and/or increased PSA value.

Bone scintigraphy can be performed by using either a planar or a tomographic (single-photon emission computed tomography [SPECT]) acquisition protocol: a non-contrastenhanced CT scan can be performed in addition to tomographic SPECT acquisition (SPECT/CT) for anatomical correlation and attenuation-correction purposes. A slight increase in sensitivity, yet without significant changes in specificity, when SPECT is performed as a completion of planar scan, has been reported. ${ }^{17}$ Since osteoblastic reaction occurs much earlier than anatomical changes in cortical bone, bone scintigraphy using MDP or HMDP is much more sensitive than such morphological modalities as CT or magnetic resonance imaging (MRI) and detects bone metastases long before anatomical changes appear. Since marked osteoblastic reaction is common in bone metastases from prostate cancer, bone scintigraphy is the most sensitive, widely available, and easy-to-perform diagnostic method in this setting, currently.

Although very sensitive, bone scintigraphy has low specificity, since both MDP and HMDP accumulate in the surrounding bone reaction, not inside the neoplastic cells, thus a higher uptake of radiopharmaceutical in a skeletal site could be ascribed to each event able to produce an increased bone apposition in that site. ${ }^{15,18}$ In a very recent prospective study, Damle et $\mathrm{al}^{19}$ evaluated 151 patients with suspected bone metastases from either lung, breast, or prostate cancers by using bone scan and PET/CT: overall specificity of bone scintigraphy (in both patients at first diagnosis and patients with suspected recurrent disease) was 54\% in lung cancer 
patients, $63 \%$ in breast cancer patients, and $41 \%$ in prostate cancer patients. Sclerotic lesions were more commonly detected than mixed lytic ones (70 vs eleven and eight, respectively). False-positive findings on bone scintigraphy are more commonly consequent to traumatic/microtraumatic injury (accidental or iatrogenic), joint degenerative or inflammatory disease (arthrosis, osteoarthritis), metabolic disorders (eg, hyperparathyroidism), or benign bone diseases (eg, benign neoplasms, Paget's disease). ${ }^{15,18}$ Moreover, as described by Withofs et al, ${ }^{20}$ the overall accuracy of bone scintigraphy varies depending on the location of findings in the skeleton, being as low as $42 \%$ and $51 \%$ for pelvis and lumbar spine, respectively; conversely, higher accuracy values are shown for skull and long-bone lesions ( $83 \%$ and $75 \%$, respectively).

Therefore, a careful collection of clinical history, paying particular attention to recent traumatic injuries and previous surgical interventions, is mandatory to reduce the risk of misinterpretation of benign findings.

\section{Positron emission tomography}

PET is a nuclear medicine diagnostic technique that uses molecules labeled with positron-emitting radionuclides, such as fluorine-18 or carbon-11, to trace physiological or pathological aspects and processes within the human body. Due to the lack of anatomical landmarks, PET is usually performed in coregistration with contrast-enhanced or more frequently non-contrast-enhanced CT: this hybrid morphological and functional technique is called PET/CT.

A number of radiopharmaceuticals are currently available for PET/CT evaluation of prostate cancer, for both initial staging and restaging purposes: detection of distant (bone and soft tissue) metastases and nodal sites of disease, rather than the depiction of the primary lesion, are the primary indications to perform a PET/CT examination in patients with recently diagnosed prostate cancer. Particularly, fluorine-18fluorodeoxyglucose (FDG), fluorine-18-fluorocholine (FCh), or carbon-11-choline (CCh) and sodium fluorine-18-fluoride $(\mathrm{NaF})$ are currently used in PET/CT investigation of bone metastases from prostate cancer.

\section{Fluorine- | 8-fluorodeoxyglucose}

FDG is a radioactive glucose analogue that is actively trapped into cells by means of transmembrane glucose transporters, whose expression on the cell membrane is physiologically variable in response to the metabolic needs of the cell itself. The rationale of using FDG to detect tumoral sites is that glucose transporters are abnormally overexpressed on the cell membrane of neoplastic cells due to dysregulation of metabolic pathways; besides, hexokinase II enzymatic activity is dramatically enhanced in tumors, so that phosphorylated FDG (as FDG-6-phosphate) accumulates into neoplastic cells much more often than into normal ones. ${ }^{21,22}$ The phenomenon of FDG uptake by prostate cancer cells is variable, mostly depending on the grade of differentiation and aggressiveness: higher FDG uptake can be observed in tumors that are resistant to androgen-deprivation therapy than in androgen-dependent ones. ${ }^{23}$

However, the few studies that have specifically investigated the efficacy of FDG PET/CT using FDG in the detection of skeletal sites from prostate cancer have demonstrated a high risk of false-negative results: low glycolytic activity of prostate cancer cells and physiological urinary excretion account for low sensitivity of PET/CT using FDG in this setting. Therefore, FDG is of little or no interest for detecting skeletal sites from prostate cancer in current clinical practice. Shreve et $\mathrm{al}^{24}$ observed that PET/CT using FDG identified only 131 out of 202 untreated skeletal metastases in 22 patients, with a sensitivity of $65 \%$, lower than that of conventional bone scintigraphy in the same population; moreover, in six patients undergoing hormonal treatment, FDG identified merely four out of 131 skeletal sites on bone scan. Yeh et $\mathrm{al}^{25}$ reported that only about $18 \%$ of skeletal sites of disease on conventional bone scan showed a corresponding increase of FDG uptake; this finding was independent of duration of illness, entity of PSA increase, previous hormone therapy, and overall extent of disease. More recently, Morris et $\mathrm{al}^{26}$ observed that despite its low sensitivity, PET/ CT using FDG could be helpful in distinguishing progressive from quiescent bone metastatic disease in patients with prostate cancer: although $\mathrm{PET/CT}$ was falsely negative in 31 out of 126 metastatic sites seen on bone scintigraphy (sensitivity 77\%), all but one lesion seen on bone scan alone (FDG-negative) showed no progression on follow-up when compared with the baseline bone scan; on the contrary, all 95 FDG-positive lesions reflected progressive disease on subsequent studies. Therefore, FDG uptake should predict further progression of skeletal disease.

In support of these observations, it has been supposed that glucose metabolism is not important in providing prostate cancer with a source of energy. Moreover, sclerotic prostate cancer metastases may be relatively hypocellular, so that viable tumor volume is under the detection limit of the PET/ CT technique; conversely, these lesions are typically positive on conventional bone scan, since the radiopharmaceutical is actively bound by osseous matrix. Osteolytic metastases, 
instead, reveal a particularly aggressive behavior of the tumor, which results in reduced blood flow, hypoxia, and enhanced glycolysis (FDG-positive lesions).

\section{Fluorine- I 8-fluorocholine and carbon-I I-choline}

Choline is an essential nutrient that acts as a substrate for the synthesis of phosphatidylcholine and sphingomyelin, two classes of cell membrane phospholipids. Their synthesis by choline kinase is upregulated in tumor cells. ${ }^{27,28}$ Ackerstaff et $\mathrm{al}^{27}$ observed that malignant prostate cells exhibit significantly higher levels of choline-derived compound compared with normal prostate epithelial and stromal cells; such behavior is attributable to an alteration of phospholipid metabolism rather than increased cell density. Therefore, choline-derived radiopharmaceuticals, such as $\mathrm{FCh}$ and $\mathrm{CCh}$, are currently the most commonly used tracers for PET/CT evaluation of prostate cancer. ${ }^{29}$ The short halflife of carbon-11 (20 minutes) requires an in-site cyclotron, and this limits the use of $\mathrm{CCh}$ in clinical practice. FCh was developed as a more versatile alternative, thanks to the longer half-life of nuclide fluorine-18 (about 110 minutes).

Currently, the primary indication for PET/CT using FCh or $\mathrm{CCh}$ is the detection of eventual sites of recurrent disease in patients with biochemical relapse (namely, a rise in PSA values regardless of concurrent hormone therapy). A recent meta-analysis by Evangelista et $\mathrm{al}^{30}$ reported high sensitivity and specificity of PET/CT using choline-derived radiopharmaceuticals ( $85.6 \%$ and $92.6 \%$, respectively) in detecting sites of prostate cancer recurrence after radical prostatectomy or external beam radiation therapy.

Several studies have investigated the reliability of $\mathrm{PET} / \mathrm{CT}$ using choline-derived radiopharmaceuticals in the detection of bone metastases from prostate cancer. Beheshti et al ${ }^{31}$ studied 38 men with biopsy-proven prostate cancer, either preoperatively or postoperatively, and evaluated both patient-based and lesion-based accuracy of PET/CT using FCh in 321 bone lesions, comparing imaging findings with histopathology and/or clinical follow-up, considered as gold-standard references: sensitivity, specificity, and overall accuracy were $74 \%, 99 \%$, and $85 \%$, respectively. Moreover, they found that FCh uptake negatively correlates with bone lesion density, expressed as Hounsfield unit (HU) level: bone marrow metastases without significant bone reaction and remodeling and lytic lesions tend to be more frequently FCh-positive, while highly sclerotic lesions with predominant osteoblastic reaction do not show significant FCh uptake. In their study, no lesions with CT density more than 825 HU were FCh-positive. Smaller numbers of cancer cells, eventually with low metabolic activity, and lower blood supply in sclerotic lesions are possible explanations of poor FCh uptake, similar to the lower FDG uptake observed in sclerotic metastases.

These encouraging results were confirmed in a wider study on 70 patients: ${ }^{32}$ sensitivity, specificity and overall accuracy values were $79 \%, 97 \%$, and $84 \%$, respectively, on a lesion-based analysis. A significant correlation was found between FCh uptake, expressed as maximum standardized uptake value ( $\left.\mathrm{SUV}_{\max }\right)$, and lesion density, expressed as $\mathrm{HU}$ level. Low FCh uptake was observed in highly sclerotic lesions, even in cases with biochemical evidence of progressive disease and in two patients undergoing hormone therapy, although there were multiple FCh-positive metastases in other skeletal sites. Moreover, PET/CT using FCh is helpful in detecting early bone marrow infiltration without evidence of morphological alterations: such FCh-positive findings lead to significant changes in the management of high-risk patients in preoperative staging. ${ }^{32,33}$ Tuncel et $\mathrm{al}^{34}$ observed in a population of 45 advanced prostate cancer patients that bone lesions with high uptake of $\mathrm{CCh}$ showed an average density of $458 \mathrm{HU}$, in comparison with $\mathrm{CCh}$ negative lesions, which showed an average density of 787 HU (sclerotic).

Finally, clinical follow-up of patients with progressive prostate cancer has revealed that bone lesions from prostate cancer usually evolve from FCh-positive/CT-negative, lowdensity, high-cellularity metastases (substantially, expression of bone marrow involvement) to usually slightly osteoblastic alterations, with mild-to-moderate positivity on FCh and positivity on CT, and finally progress to high-density, sclerotic FCh-negative lesions, with a limited amount of viable cancer cells. ${ }^{32}$ Furthermore, as reported by Beheshti et al, ${ }^{32}$ hormone therapy tends to increase average density of bone lesions (713 HU in previously treated vs $542 \mathrm{HU}$ in untreated patients), despite no significant changes on average $\mathrm{SUV}_{\text {max }}$ (8.2 vs 7.9), presumably as a consequence of posttreatment apoptotic phenomena.

Lastly, a recent meta-analytic paper by Umbehr et al ${ }^{35}$ investigated the role of both $\mathrm{FCh}$ and $\mathrm{CCh}$ in staging patients with proven but untreated prostate cancer: sensitivity, specificity and diagnostic odds ratio were $84 \%, 79 \%$, and $20 \%$, respectively, on a per-patient basis, and $66 \%, 92 \%$, and $23 \%$, respectively, on a per-lesion basis. The authors underlined that there is limited but promising evidence that PET/CT using FCh or CCh performs well in this setting, warranting further studies. 
Rare variants of prostate carcinoma, such as smallcell subtype, should be considered as a possible source of false-negative findings on PET/CT using choline-derived radiopharmaceuticals. ${ }^{36}$ False-positive findings are usually related to nonspecific uptake of $\mathrm{FCh}$ or $\mathrm{CCh}$ by inflammatory cells; this could result in significant uptake by both actively remodeling pagetic bone and coexisting skeletal metastases from prostate cancer, as reported by Giovacchini et al. ${ }^{37}$

\section{Sodium fluorine- | 8-fluoride}

$\mathrm{NaF}$ is a sodium salt of fluorine-18 that binds to calcium ions in hydroxyapatite crystals in bone. Therefore, it is successfully used as a PET/CT bone-seeking tracer to detect skeletal abnormalities, mainly for oncological purposes (detection of distant bone metastases): uptake of $\mathrm{NaF}$ reflects blood flow and bone remodeling, which are increased in sites of metastatic spread to the bone. Greater accuracy values than those observed in radionuclide bone scintigraphy using technetium-99m-labeled samples have been reported in the detection of skeletal metastases from different primary tumors. ${ }^{20,38-40}$ It has been demonstrated not only that PET/ $\mathrm{CT}$ using $\mathrm{NaF}$ is highly reliable in detecting both sclerotic and osteolytic lesions and in differentiating malignant from benign lesions but also that it is more accurate than bone scintigraphy, especially in spine and pelvic lesions..$^{38-41}$ A meta-analysis by Tateishi et al ${ }^{42}$ confirmed sensitivity and specificity values of $96.2 \%$ and $98.5 \%$, respectively, on a patient basis, and $96.9 \%$ and $98.0 \%$, respectively, on a lesion basis.

Many studies have investigated the role of PET/CT using $\mathrm{NaF}$ in patients with prostate cancer. Even-Sapir et a ${ }^{17}$ found that PET using $\mathrm{NaF}$ is a very sensitive tool for the detection of bone metastases in patients with high-risk prostate cancer, with reported sensitivity, specificity, and positive and negative predictive values of $100 \%, 62 \%, 74 \%$, and $100 \%$, respectively. In particular, 21 patients with negative $\mathrm{NaF}$ PET/CT had no clinical or imaging evidence of metastatic spread for at least a 6-month follow-up period. The specificity of $\mathrm{NaF}$ is greater than that of bone scintigraphy, especially in depicting malignant lesions rather than benign ones; besides, the addition of CT scan to PET alone contributed to an increase in diagnostic accuracy and specificity, due to a possible correlation of function changes with morphological alterations.

Beheshti et $a l^{31}$ evaluated 321 bone lesions from prostate cancer and found that $\mathrm{NaF}$ is more sensitive than $\mathrm{FCh}$ ( $81 \%$ vs $74 \%$ ); however, a significant change in patients' management was not observed in comparison with FCh.
Furthermore, osteoblastic lesions usually showed intense $\mathrm{NaF}$ uptake due to increased apposition of mineralized matrix; conversely, possible negative findings have been reported in highly sclerotic dense lesions in patients undergoing antiandrogenic therapy, as a consequence of effective treatment.

More recently, Damle et $a{ }^{19}$ prospectively studied 49 prostate cancer patients ( 25 for initial staging and 24 for restaging purposes) without known bone metastases but with high risk/clinical suspicion (eg, bone pain). The authors found that PET/CT using NaF had the highest possible sensitivity and negative predictive value $(100 \%)$ and $90 \%$ overall accuracy, when compared with FDG and bone scintigraphy. Particularly, PET/CT using NaF detected the greatest number of lesions, followed by bone scintigraphy, followed in turn by FDG. Therefore, they inferred that PET/CT using $\mathrm{NaF}$ is of the greatest importance in patients with high risk or high clinical suspicion for bone metastases from either prostate or breast and lung cancers (the authors also studied 72 and 30 patients with breast and lung carcinomas, respectively).

Withofs et al ${ }^{20}$ prospectively investigated the accuracy of PET/CT using $\mathrm{NaF}$ in ten patients with prostate cancer and at high risk of bone metastases, using MRI or thin-slice CT as gold-standard imaging methods. All patients were also studied with both planar and SPECT bone scintigraphy using technetium-99m-labeled bisphosphonates. PET/CT using $\mathrm{NaF}$ was confirmed to be more accurate than bone scintigraphy in detecting skeletal metastases from prostate cancer: sensitivity, specificity, accuracy, and positive and negative predictive values were $100 \%, 95 \%, 96 \%, 86 \%$, and $100 \%$, respectively (vs $67 \%, 84 \%, 80 \%, 57 \%$, and $89 \%$ for bone scintigraphy), for lesions confirmed as malignant, and $100 \%, 89 \%, 92 \%, 75 \%$, and $100 \%$, respectively (vs $67 \%, 82 \%, 78 \%, 53 \%$ and $89 \%$ for bone scintigraphy), for benign findings. False-positive findings were mostly due to posttrauma osteoblastic activity for both $\mathrm{NaF}$ and bone scintigraphy. Tomographic acquisition did not significantly contribute to overall accuracy of planar bone scintigraphy. The accuracy of NaF was significantly higher than bone scintigraphy for pelvic and lumbar spine malignant lesions ( $73 \%$ vs $42 \%$ and $78 \%$ vs $51 \%$, respectively), rather than for lesions located in other skeletal sites. The sensitivity of $\mathrm{NaF}$ and bone scintigraphy were related to heterogeneity of presentation of bone metastases, with greater accuracy in osteoblastic lesions.

The detection of bone metastases by using $\mathrm{NaF}$ and $\mathrm{FCh}$ in 42 patients with prostate cancer complaining of osteoarticular pain was evaluated prospectively by Langsteger et al. ${ }^{43}$ Despite overall similar sensitivity between $\mathrm{NaF}$ and FCh in 
360 skeletal lesions (91\%), FCh showed greater overall specificity (89\% vs $83 \%$ ) and accuracy (90\% vs 88\%; however, no statistical significance was reached in this setting). By considering only patients at initial staging, no significant differences were seen between FCh and NaF in site-based performance.

\section{Clinical recommendations}

The role of nuclear medicine imaging tools in evaluating patients with biochemical recurrence of prostate cancer (ie, increase in PSA levels) after radical treatment has been extensively investigated. In particular, PET/CT using FCh has shown high reliability in the detection of both locoregional and distant metastases in patients with biochemical relapse..$^{30}$ Conversely, the proper use of imaging techniques in the initial staging of patients with recently diagnosed prostate cancer is more controversial.

Early detection of distant metastases, and particularly skeletal ones, is useful in the correct staging, definition of prognosis, and further treatment decision-making for patients with recently diagnosed prostate cancer; in fact, patients with extensive skeletal disease may benefit from systemic therapies, such as androgen deprivation or bisphosphonates, while those with localized disease may take advantage of surgical and/or radiation therapy. ${ }^{13,14}$ However, the routine use of skeletal imaging is not recommended in all patients for staging purposes. The Best Practice Statement edited in 2000 by the American Urological Association and recently updated in 2009 recommended pretreatment staging of prostate cancer only in cases of high-risk disease: PSA level, GS at biopsy, and clinical stage of disease could be used to predict the yield of imaging in patients' further management. ${ }^{44-47}$ Since pretreatment serum PSA level correlates with the risk of extraprostatic extension, routine bone scan is not required for staging asymptomatic patients with clinically localized prostate cancer when PSA level is equal to or less than $20 \mathrm{ng} / \mathrm{mL}$, unless the history or clinical examination suggests skeletal involvement. Abuzallouf et $\mathrm{al}^{47}$ reported that metastases were found on bone scans in $5.3 \%$ of patients with PSA levels lower than $20 \mathrm{ng} / \mathrm{mL}$; conversely, 16.2\% of patients with PSA levels higher than $20 \mathrm{ng} / \mathrm{mL}$ showed bone metastases. Higher GS $(>7)$ at biopsy significantly correlates with higher risk of bone involvement, as recently reported by De Nunzio et al. ${ }^{48}$

Despite well-defined clinical recommendations, widespread overuse of imaging in low-risk and intermediaterisk prostate cancer patients has been reported. Expensive unnecessary imaging is common in older and less educated people, and in patients managed with proton-beam or standard radiation therapy rather than in those undergoing radical prostatectomy or active surveillance. ${ }^{49,50}$ Anyway, staging procedures that are not expected to affect further treatment decision-making should be avoided.

To date, bone scintigraphy using MDP or HMDP has been the cornerstone in the evaluation of newly diagnosed high-risk prostate cancer patients, since it is a widely available and inexpensive whole-body imaging technique that allows the reliable ruling out of skeletal involvement in a single examination, due to its high sensitivity. However, this technique suffers from low specificity and is not able to exclude osteolytic and soft-tissue metastases. Therefore, PET/CT using choline-derived radiopharmaceuticals is used even more in this setting to detect both soft-tissue and skeletal metastases (Figure 1); indeed, it has been proposed as a "one-stop shop" technique in the evaluation of metastases from prostate cancer, due to its higher specificity. However, there is little agreement in the literature about which method is the best, since discrepancies between PET/CT using FCh or $\mathrm{CCh}$ and bone scintigraphy have been described. ${ }^{51-54}$ However, as recently reported by Balogova et al, ${ }^{55}$ generalized skeletal uptake in patients receiving bone marrowstimulating factors (like erythropoietin) could reduce the sensitivity of PET/CT using FCh or CCh; such behavior is not seen in bone-seeking radiopharmaceuticals, such as $\mathrm{NaF}$ and bisphosphonates. Another well-known drawback of PET/CT using choline-derived radiopharmaceuticals is its low negative predictive value, mainly due to its limited capability to detect micrometastases. ${ }^{56,57}$

PET/CT using NaF should be preferred to PET/CT using $\mathrm{FCh}$ or $\mathrm{CCh}$ in the evaluation of patients with high-density sclerotic bone metastases, due to the naturally increased uptake of $\mathrm{NaF}$ in sites of bone remodeling; moreover, better spatial resolution of PET modality and the advantages deriving from tomographic acquisition and CT-dependent attenuation correction allow $\mathrm{NaF}$ to perform better than classic bone scintigraphy in the depiction of small skeletal sites of disease and in mixed-type lesions. Its very high sensitivity and negative predictive value make it very useful in detecting occult skeletal metastases in patients with biochemical relapse, and positivity tends to associate with increasing PSA levels. ${ }^{58}$ Higher specificity than diffusion-weighted MRI has recently been reported. ${ }^{59}$ However, further studies comparing these techniques and correlating imaging findings with PSA values and PSA velocity are needed.

The role of PET/CT using FDG is not well defined in this setting, since a very low detection rate of bone and soft-tissue 

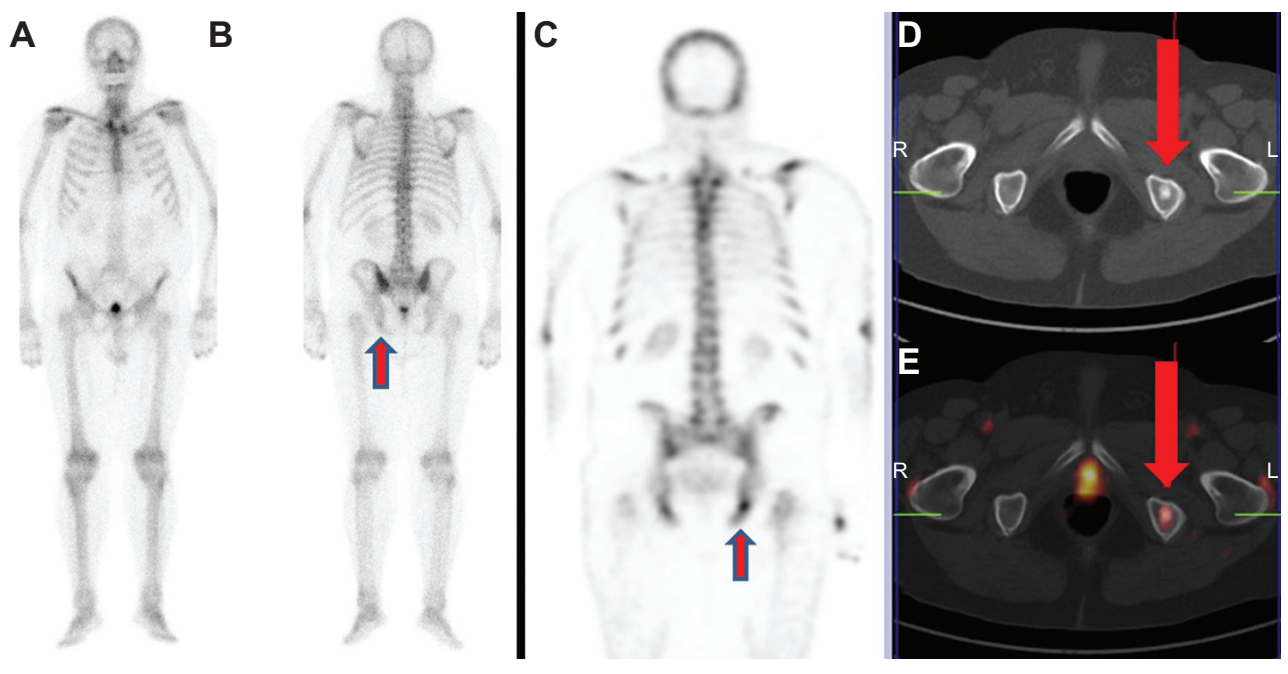

Figure I (A-E) A 76-year-old patient with recently diagnosed prostate cancer (prostate-specific antigen level, $18 \mathrm{ng} / \mathrm{mL}$ ) underwent bone scan and positron emission tomography (PET)/computed tomography (CT) using radiolabeled choline for staging purposes. Bone scan in anterior (A) and posterior view (B) shows an area of mild radiopharmaceutical uptake corresponding to the left ischiatic bone (arrow). This finding was more evident by using single-photon emission computed tomography imaging (C).

Notes: Axial CT (D) and radiolabeled choline PET/CT (E) images showed the presence of increased radiopharmaceutical uptake corresponding to an osteosclerotic lesion located in the left ischiatic bone (arrows). This finding was highly suspicious for skeletal metastasis. Biopsy confirmed the presence of a bone metastasis by prostate cancer.

metastases is commonly described. As reported by Jadvar in a recent review article, ${ }^{60}$ FDG uptake in prostate cancer depends on tumor differentiation, with low accumulation in well-differentiated tumors and high uptake in aggressive poorly differentiated tumors.

\section{Conclusion}

Radionuclide bone scintigraphy using labeled bisphosphonates, either performed with planar, SPECT, or SPECT/CT acquisition, remains the most widely available and simple technique for the early detection of bone metastases in prostate cancer patients at diagnosis. However, PET/CT using different non-FDG radiopharmaceuticals ( $\mathrm{FCh}, \mathrm{CCh}$, and $\mathrm{NaF}$ ) is emerging as a possible alternative to bone scintigraphy in this setting. Indeed, choline-derived radiopharmaceuticals are helpful in the detection of small lytic skeletal metastases, nodal and soft-tissue metastases, despite low sensitivity in the evaluation of osteoblastic lesions; on the other hand, $\mathrm{NaF}$ shows high sensitivity (due to the detection of even minimal changes in the bone turnover and blood flow in metastatic sites), overcoming the low specificity of bone scintigraphy, thanks to its higher spatial resolution and the anatomical correlate deriving from CT scan. Greater radiobiological burden, higher costs, and the restricted territorial availability of PET/CT methods, however, limit their use as first-line investigations in patients with prostate cancer.

\section{Disclosure}

The authors report no conflicts of interest in this work.

\section{References}

1. Brawley OW. Prostate cancer epidemiology in the United States. World J Urol. 2012;30(2):195-200.

2. Crawford ED. Epidemiology of prostate cancer. Urology. 2003; 62(6 Suppl 1):3-12.

3. Punnen S, Cooperberg MR. The epidemiology of high-risk prostate cancer. Curr Opin Urol. Epub April 24, 2013.

4. Soloway MS, Hardeman SW, Hickey D, et al. Stratification of patients with metastatic prostate cancer based on extent of disease on initial bone scan. Cancer. 1988;61(1):195-202.

5. Ohmori K, Matsui H, Yasuda T, et al. Evaluation of the prognosis of cancer patients with metastatic bone tumors based on serial bone scintigrams. Jpn J Clin Oncol. 1997;27(4):263-267.

6. Sabbatini P, Larson SM, Kremer A, et al. Prognostic significance of extent of disease in bone in patients with androgen-independent prostate cancer. J Clin Oncol. 1999;17(3):948-957.

7. Rigaud J, Tiguert R, Le Normand L, et al. Prognostic value of bone scan in patients with metastatic prostate cancer treated initially with androgen deprivation therapy. $J$ Urol. 2002;168(4 Pt 1): 1423-1426.

8. Cheng HH, Lin DW, Yu EY. Advanced clinical states in prostate cancer. Urol Clin North Am. 2012;39(4):561-571.

9. Tanaka N, Fujimoto K, Shinkai T, et al. Bone scan can be spared in asymptomatic prostate cancer patients with PSA of $<=20 \mathrm{ng} / \mathrm{mL}$ and Gleason score of $<=6$ at the initial stage of diagnosis. Jpn J Clin Oncol. 2011;41(10):1209-1213.

10. Moslehi M, Cheki M, Salehi-Marzijarani M, Amuchastegui T, Gholamrezanezhad A. Predictors of bone metastasis in pre-treatment staging of asymptomatic treatment-naïve patients with prostate cancer. Rev Esp Med Nucl Imagen Mol. Epub March 8, 2013.

11. Long MA, Husband JE. Features of unusual metastases from prostate cancer. Br J Radiol. 1999;72(862):933-941.

12. Reigman HI, Stokkel MP. Peripheral bone metastases in prostate cancer: a rare localization at initial presentation. Clin Nucl Med. 2004;29(5):335-336.

13. Tombal B, Lecouvet F. Modern detection of prostate cancer's bone metastasis: is the bone scan era over? Adv Urol. 2012;2012:893193.

14. Horwich A, Hugosson J, de Reijke T, Wiegel T, Fizazi K, Kataja V. Prostate cancer: ESMO Consensus Conference Guidelines 2012. Ann Oncol. 2013;24(5):1141-1162. 
15. Mari Aparici C, Seo Y. Functional imaging for prostate cancer: therapeutic implications. Semin Nucl Med. 2012;42(5):328-342.

16. Khokher MA, Dandona P. Diphosphonates inhibit human osteoblast secretion and proliferation. Metabolism. 1989;38(2):184-187.

17. Even-Sapir E, Metser U, Mishani E, Lievshitz G, Lerman H, Leibovitch $\mathrm{I}$. The detection of bone metastases in patients with highrisk prostate cancer: $99 \mathrm{mTc}-\mathrm{MDP}$ planar bone scintigraphy, single- and multi-field-of-view SPECT, 18F-fluoride PET, and 18F-fluoride PET/ CT. J Nucl Med. 2006;47(2):287-297.

18. Langsteger $\mathrm{W}$, Haim $\mathrm{S}$, Knauer $\mathrm{M}$, et al. Imaging of bone metastases in prostate cancer: an update. Q J Nucl Med Mol Imaging. 2012;56(5): $447-458$.

19. Damle NA, Bal C, Bandopadhyaya GP, et al. The role of (18)F-fluoride PET-CT in the detection of bone metastases in patients with breast, lung and prostate carcinoma: a comparison with FDG PET/CT and (99m) Tc-MDP bone scan. Jpn J Radiol. 2013;31(4):262-269.

20. Withofs N, Grayet B, Tancredi T, et al. ${ }^{18} \mathrm{~F}$-fluoride PET/CT for assessing bone involvement in prostate and breast cancers. Nucl Med Commun. 2011;32(3):168-176.

21. Macheda ML, Rogers S, Best JD. Molecular and cellular regulation of glucose transporter (GLUT) proteins in cancer. J Cell Physiol. 2005;202(3):654-662.

22. Smith TA. Mammalian hexokinases and their abnormal expression in cancer. Br J Biomed Sci. 2000;57(2):170-178.

23. Jadvar H, Xiankui L, Shahinian A, et al. Glucose metabolism of human prostate cancer mouse xenografts. Mol Imaging. 2005;4(2):91-97.

24. Shreve PD, Grossman HB, Gross MD, Wahl RL. Metastatic prostate cancer: initial findings of PET with 2-deoxy-2-[F-18]fluoro-D-glucose. Radiology. 1996;199(3):751-756.

25. Yeh SD, Imbriaco M, Larson SM, et al. Detection of bony metastases of androgen-independent prostate cancer by PET-FDG. Nucl Med Biol. 1996;23(6):693-697.

26. Morris MJ,Akhurst T, Osman I, et al. Fluorinated deoxyglucose positron emission tomography imaging in progressive metastatic prostate cancer. Urology. 2002;59(6):913-918.

27. Ackerstaff E, Pflug BR, Nelson JB, Bhujwalla ZM. Detection of increased choline compounds with proton nuclear magnetic resonance spectroscopy subsequent to malignant transformation of human prostatic epithelial cells. Cancer Res. 2001;61(9):3599-3603.

28. Sutinen E, Nurmi M, Roivainen A, et al. Kinetics of [(11)C]choline uptake in prostate cancer: a PET study. Eur J Nucl Med Mol Imaging. 2004;31(3):317-324.

29. Bouchelouche K, Tagawa ST, Goldsmith SJ, Turkbey B, Capala J, Choyke P. PET/CT imaging and radioimmunotherapy of prostate cancer. Semin Nucl Med. 2011;41(1):29-44.

30. Evangelista L, Zattoni F, Guttilla A, et al. Choline PET or PET/CT and biochemical relapse of prostate cancer: a systematic review and metaanalysis. Clin Nucl Med. 2013;38(5):305-314.

31. Beheshti M, Vali R, Waldenberger P, et al. Detection of bone metastases in patients with prostate cancer by $18 \mathrm{~F}$ fluorocholine and $18 \mathrm{~F}$ fluoride PET-CT: a comparative study. Eur J Nucl Med Mol Imaging. 2008; 35(10): 1766-1774.

32. Beheshti M, Vali R, Waldenberger P, et al. The use of F-18 choline PET in the assessment of bone metastases in prostate cancer: correlation with morphological changes on CT. Mol Imaging Biol. 2010;12(1):98-107.

33. Beheshti M, Imamovic L, Broinger G, et al. $18 \mathrm{~F}$ choline PET/CT in the preoperative staging of prostate cancer in patients with intermediate or high risk of extracapsular disease: a prospective study of 130 patients. Radiology. 2010;254(3):925-933.

34. Tuncel M, Souvatzoglou M, Herrmann K, et al. [(11)C]Choline positron emission tomography/computed tomography for staging and restaging of patients with advanced prostate cancer. Nucl Med Biol. 2008;35(6):689-695.

35. Umbehr MH, Müntener M, Hany T, Sulser T, Bachmann LM. The role of $11 \mathrm{C}$-choline and $18 \mathrm{~F}$-fluorocholine positron emission tomography (PET) and PET/CT in prostate cancer: a systematic review and metaanalysis. Eur Urol. Epub April 19, 2013.
36. Al-Tamimi A, Tan AE, Kwong SY, Sam CC, Chong A, Tan CH. Falsenegative bone scan and choline PET/CT study in a case of prostate cancer: the pitfall of the small cell prostate carcinoma variant. World $J$ Nucl Med. 2012;11(2):75-78.

37. Giovacchini G, Gajate AM, Messa C, Fazio F. Increased C-11 choline uptake in pagetic bone in a patient with coexisting skeletal metastases from prostate cancer. Clin Nucl Med. 2008;33(11):797-798.

38. Schirrmeister H, Guhlmann A, Elsner K, et al. Sensitivity in detecting osseous lesions depends on anatomic localization: planar bone scintigraphy versus 18F PET. J Nucl Med. 1999;40(10):1623-1629.

39. Hetzel M, Arslandemir C, König HH, et al. F-18 NaF PET for detection of bone metastases in lung cancer: accuracy, cost-effectiveness, and impact on patient management. J Bone Miner Res. 2003;18(12): 2206-2214.

40. Krüger S, Buck AK, Mottaghy FM, et al. Detection of bone metastases in patients with lung cancer: $99 \mathrm{mTc}-\mathrm{MDP}$ planar bone scintigraphy, 18F-fluoride PET or 18F-FDG PET/CT. Eur J Nucl Med Mol Imaging. 2009;36(11):1807-1812.

41. Li Y, Schiepers C, Lake R, Dadparvar S, Berenji GR. Clinical utility of (18)F-fluoride PET/CT in benign and malignant bone diseases. Bone. 2012;50(1):128-139.

42. Tateishi U, Morita S, Taguri M, et al. A meta-analysis of (18)F-fluoride positron emission tomography for assessment of metastatic bone tumor. Ann Nucl Med. 2010;24(7):523-531.

43. Langsteger W, Balogova S, Huchet V, et al. Fluorocholine (18F) and sodium fluoride (18F) PET/CT in the detection of prostate cancer: prospective comparison of diagnostic performance determined by masked reading. Q J Nucl Med Mol Imaging. 2011;55(4):448-457.

44. [No authors listed]. Prostate-specific antigen (PSA) best practice policy. American Urological Association (AUA). Oncology (Williston Park). 2000;14(2):267-272, 277-278, 280 passim.

45. American Urological Association. Prostate-specific antigen (PSA) best practice statement: 2009 update. Available from: http://www.auanet.org/ content/guidelines-and-quality-care/clinical-guidelines/main-reports/ psa09.pdf. Accessed April 23, 2013.

46. Greene KL, Albertsen PC, Babaian RJ, et al. Prostate specific antigen best practice statement: 2009 update. J Urol. 2013;189(Suppl 1): S2-S11.

47. Abuzallouf S, Dayes I, Lukka H. Baseline staging of newly diagnosed prostate cancer: a summary of the literature. J Urol. 2004;171(6 Pt 1): 2122-2127.

48. De Nunzio C, Leonardo C, Franco G, et al. When to perform bone scan in patients with newly diagnosed prostate cancer: external validation of a novel risk stratification tool. World J Urol. 2013;31(2):365-369.

49. Choi WW, Williams SB, Gu X, Lipsitz SR, Nguyen PL, Hu JC. Overuse of imaging for staging low risk prostate cancer. $J$ Urol. 2011;185(5): $1645-1649$.

50. Prasad SM, Gu X, Lipsitz SR, Nguyen PL, Hu JC. Inappropriate utilization of radiographic imaging in men with newly diagnosed prostate cancer in the United States. Cancer. 2012;118(5):1260-1267.

51. Garcia G Jr, Riera E, Valls E, et al. Bone metastases from prostate cancer: 18F-fluoride PET/CT in a patient with discordant bone scintigraphy and 11C-choline PET/CT. Clin Nucl Med. 2013;38(2):120-124.

52. Fuccio C, Castellucci P, Schiavina R, et al. Role of 11C-choline PET/ $\mathrm{CT}$ in the re-staging of prostate cancer patients with biochemical relapse and negative results at bone scintigraphy. Eur J Radiol. 2012;81(8): e893-e896.

53. Picchio M, Spinapolice EG, Fallanca F, et al. [11C]Choline PET/ CT detection of bone metastases in patients with PSA progression after primary treatment for prostate cancer: comparison with bone scintigraphy. Eur J Nucl Med Mol Imaging. 2012;39(1):13-26.

54. Fuccio C, Castellucci P, Schiavina R, et al. Role of 11C-choline PET/ $\mathrm{CT}$ in the restaging of prostate cancer patients showing a single lesion on bone scintigraphy. Ann Nucl Med. 2010;24(6):485-492.

55. Balogova S, Huchet V, Egrot C, et al. Effect of erythropoietin on bone marrow uptake of $18 \mathrm{~F}$-fluorocholine in prostate cancer: comparison with 18F-fluoride uptake. Clin Nucl Med. 2013;38(3):200-202. 
56. Picchio M, Castellucci P. Clinical indications of C-choline PET/CT in prostate cancer patients with biochemical relapse. Theranostics. 2012;2(3):313-317.

57. Scattoni V, Picchio M, Suardi N, et al. Detection of lymph-node metastases with integrated [11C]choline PET/CT in patients with PSA failure after radical retropubic prostatectomy: results confirmed by open pelvic-retroperitoneal lymphadenectomy. Eur Urol. 2007;52(2): 423-429.

58. Jadvar H, Desai B, Ji L, et al. Prospective evaluation of $18 \mathrm{~F}-\mathrm{NaF}$ and 18 F-FDG PET/CT in detection of occult metastatic disease in biochemical recurrence of prostate cancer. Clin Nucl Med. 2012;37(7): $637-643$.
59. Mosavi F, Johansson S, Sandberg DT, et al. Whole-body diffusionweighted MRI compared with (18)F-NaF PET/CT for detection of bone metastases in patients with high-risk prostate carcinoma. AJR Am J Roentgenol. 2012;199(5):1114-1120.

60. Jadvar H. Imaging evaluation of prostate cancer with (18) F-fluorodeoxyglucose PET/CT: utility and limitations. Eur J Nucl Med Mol Imaging. Epub February 22, 2013.

\section{Publish your work in this journal}

Cancer Management and Research is an international, peer-reviewed open access journal focusing on cancer research and the optimal use of preventative and integrated treatment interventions to achieve improved outcomes, enhanced survival and quality of life for the cancer patient The journal welcomes original research, clinical \& epidemiological studies, reviews \& evaluations, guidelines, expert opinion \& commentary, case reports \& extended reports. The manuscript management system is completely online and includes a very quick and fair peerreview system, which is all easy to use. Visit http://www.dovepress.com/ testimonials.php to read real quotes from published authors.

Submit your manuscript here: http://www.dovepress.com/cancer-management-and-research-journal 